\title{
ON THE CONVERGENCE OF BEST UNIFORM DEVIATIONS
}

\author{
BY \\ S. J. POREDA
}

\begin{abstract}
If a function $f$ is continuous on a closed Jordan curve $\Gamma$ and meromorphic inside $\Gamma$, then the polynomials of best uniform approximation to $f$ on $\Gamma$ converge interior to $\Gamma$. Furthermore, the limit function can in each case be explicitly determined in terms of the mapping function for the interior of $\Gamma$. Applications and generalizations of this result are also given.
\end{abstract}

1. Introduction. For a continuous complex valued function $f$ defined on a set $E$ in the plane let $\|f\|_{E}=\sup _{z \in E}|f(z)|$. Also, if $E$ is compact, for $n \in Z^{+}$let $p_{n}(f, E)$ denote the polynomial of degree $n$ of best uniform approximation to $f$ on E.

In general, the difference $\left[f(z)-p_{n}(f, E)(z)\right]$ does not converge to zero on $E$, and so one is naturally led to the question of whether or not this difference converges for each $z \in E$ in general. One might also inquire as to the convergence or divergence of the sequence $\left\{\left[f(z)-p_{n}(f, E)(z)\right] /\left\|f-p_{n}(f, E)\right\|_{E}\right\}$, which is of course a broader question.

By expanding on the work of Carathéodory and Fejér [1], Schur and Goluzin [2, p. 497], we are enabled to show that if $f$ is continuous on $\Gamma$, a closed Jordan curve, and meromorphic in the interior of $\Gamma$, then the sequence $\left\{f-p_{n}(f, \Gamma)\right\}_{n=0}^{\infty}$ converges in the interior of $\Gamma$ in the following sense. If $\left\{a_{i}\right\}_{i=1}^{m}$ are the poles of $f$ in the interior of $\Gamma$ with respective multiplicities $\left\{l_{i}\right\}_{i=1}^{m}$, then the sequence

$$
\left\{\prod_{i=1}^{m}\left(\frac{\phi(z)-a_{i}}{1-\bar{a}_{i} \phi(z)}\right)^{l_{i}}\left[f(z)-p_{n}(f, \Gamma)(z)\right]\right\}_{n=0}^{\infty}
$$

converges uniformly on compact subsets of the interior of $\Gamma$, where $w=\phi(z)$ is an analytic function that maps the interior of $\Gamma$ onto the open unit disc. Furthermore, we show that the limit function for the latter sequence can be explicitly determined in terms of the function $\phi$.

2. Main theorem. We will first state our main theorem in the case where $\Gamma$ is the unit circle $U$, and then in $\S 6$ present the general case. Let $D$ denote the interior of $U$. If $f$ is as above, we can write

$$
f(z)=\sigma(z) / \prod_{i=1}^{m}\left(z-a_{i}\right)^{l_{i}},
$$

where $\sigma$ is analytic in $D$ and continuous in $D+U$, and $\sigma\left(a_{i}\right) \neq 0$ for $i=1$, $2, \ldots, m$.

Received by the editors February 4, 1972.

AMS (MOS) subject classifications (1970). Primary 41A10, 41A50; Secondary 65D05, 65D15.

Key words and phrases. Best uniform approximation, closed Jordan curve. 
Theorem 1. Let $f$ be as above; then for $z \in D$,

$$
\lim _{n \rightarrow \infty}\left[f(z)-p_{n}(f, U)(z)\right] \prod_{i=1}^{m}\left(\frac{z-a_{i}}{1-\bar{a}_{i} z}\right)^{l_{i}}=\frac{1}{\lambda} \prod_{j=1}^{K}\left(\frac{z-c_{j}}{1-\bar{c}_{j} z}\right),
$$

where $\lambda$ is a prescribed root of an explicitly determined polynomial, $K \leqq\left(\sum_{i=1}^{m} l_{i}\right)$ -1 , and the $c_{j}$ 's, $j=1,2, \ldots, K$, can be explicitly determined in terms of $\lambda$. Furthermore, the convergence is uniform on compact subsets of $D$.

Remark. Whether or not (1) holds for $z \in U$ is unknown. The few examples of best approximation to meromorphic functions on $U$ that are known [5] indicate uniform convergence on $U$.

It should also be remarked that if $f$ is as in Theorem 1, we can now calculate $\lim _{n \rightarrow \infty}\left\|f-p_{n}(f, U)\right\|_{U}$, and we can find (using Taylor series for instance) a sequence of polynomials $\left\{q_{n}\right\}_{n=0}^{\infty}$ such that

$$
\lim _{n \rightarrow \infty}\left\|f-q_{n}\right\|_{U}=\lim _{n \rightarrow \infty}\left\|f-p_{n}(f, U)\right\|_{U} .
$$

3. Preliminary results. We shall first show that if $f$ is as in Theorem 1 , then there exists a unique function $g$, analytic in $D$, which minimizes

$$
\left\|[f(z)-g(z)] \prod_{i=1}^{m}\left(\frac{z-a_{i}}{1-\bar{a}_{i} z}\right)^{l_{i}}\right\|_{D}
$$

or more precisely, which minimizes

$$
\left\|f_{1}(z)-g(z) \prod_{i=1}^{m}\left(\frac{z-a_{i}}{1-\bar{a}_{i} z}\right)^{l_{i}}\right\|_{D}
$$

where $f_{1}(z)=\sigma(z) / \prod_{i=1}^{m}\left(1-\bar{a}_{i} z\right)^{l_{i}}$. We shall then show that

$$
g(z)=\lim _{n \rightarrow \infty} p_{n}(f, U)(z) \text { for } z \in D .
$$

To this end we note that proving the existence and uniqueness of such a function $g$ is equivalent to proving the existence and uniqueness of a function $F$ analytic in $D$, with the prescribed values $F^{(j)}\left(a_{i}\right)=f_{1}^{(j)}\left(a_{i}\right)$, for $i=1,2, \ldots, m$ and $j=0,1, \ldots, l_{i}-1$, and which minimizes $\|F\|_{D}$. For if $F$ is such a function we can write

$$
F(z)=f_{1}(z)-g(z) \prod_{i=1}^{m}\left(\frac{z-a_{i}}{1-\bar{a}_{i} z}\right)^{l_{i}},
$$

where $g$ is analytic in $D$. Should there exist a function $g_{1}$ analytic in $D$ for which

$$
\left\|f_{1}(z)-g_{1}(z) \prod_{i=1}^{m}\left(\frac{z-a_{i}}{1-\bar{a}_{i} z}\right)^{l_{i}}\right\|_{D}<\left\|f_{1}(z)-g(z) \prod_{i=1}^{m}\left(\frac{z-a_{i}}{1-\bar{a}_{i} z}\right)^{l_{i}}\right\|_{D}
$$


then let

$$
F_{1}(z)=f_{1}(z)-g_{1}(z) \prod_{i=1}^{m}\left(\frac{z-a_{i}}{1-\bar{a}_{i} z}\right)^{l_{i}}
$$

The function $F_{1}$ will then be analytic in $D$. Assume the values $F_{1}^{(j)}\left(a_{i}\right)=f_{1}^{(j)}\left(a_{i}\right)$ for $i=1,2, \ldots, m$ and $j=0,1, \ldots, l_{i}-1$, and $\left\|F_{1}\right\|_{D}<\|F\|_{D}$, thus contradicting our assumption about $F$. Conversely, if we start with a "minimal" $g$ and define $F$ by (2), we can likewise show that $F$ has the desired minimal property.

We shall, therefore, presently concern ourselves with the problem of determining the function $F$. We begin by establishing its existence.

Lemma 1. Let $\left\{a_{i}\right\}_{i=1}^{m}$ and $\left\{l_{i}\right\}_{i=1}^{m}$ be as in Theorem 1 and $\left\{B_{i}^{(j)}\right\}_{i=1 ; j=0}^{i=m ; j=l_{i}-1}$ be any set of $\sum_{i=1}^{m} l_{i}$ constants. Then there exists a function $F$ analytic in $D$ with $F^{(j)}\left(a_{i}\right)=B_{i}^{(j)}$ for $i=1,2, \ldots, m$ and $j=0,1, \ldots, l_{i}-1$ which minimizes $\|F\|_{D}$ for all such functions.

Proof. Clearly, the class of all such functions is nonempty. In fact, one can easily construct a polynomial $P$, which interpolates the given values and so since $\|P\|_{D}<\infty$, a straightforward application of Montel's compactness criterion [3, Vol. I, p. 415] establishes our lemma.

The following theorem completely describes the function $F$.

Theorem 2. Let $f_{1}$ be as before; then there exists a unique function $F$ analytic in D such that

$$
F^{(j)}\left(a_{i}\right)=f_{Y}^{(j)}\left(a_{i}\right) \text { for } i=1,2, \ldots, m \text { and } j=0,1, \ldots, l_{i}-1
$$

and such that $\|F\|_{D}$ is a minimum. Furthermore,

$$
F(z)=\frac{1}{\lambda} \prod_{j=1}^{K}\left(\frac{z-c_{j}}{1-\bar{c}_{j} z}\right)
$$

where $\lambda$ and the $c_{j}$ 's are as in Theorem 1 .

Corollary 1. As an immediate consequence of the above theorem, we have that there exists a unique function $g$ analytic in $D$ which minimizes

$$
\left\|f_{1}(z)-g(z) \prod_{i=1}^{m}\left(\frac{z-a_{i}}{1-\bar{a}_{i} z}\right)^{l_{i}}\right\|_{D} .
$$

Furthermore,

$$
g(z)=f(z)-\frac{1}{\lambda}\left[\prod_{i=1}^{m}\left(\frac{1-\bar{a}_{i} z}{z-a_{i}}\right)^{l_{i}}\right]\left[\prod_{j=1}^{K}\left(\frac{z-c_{j}}{1-\bar{c}_{j} z}\right)\right]
$$


4. Proof of Theorem 2. As we shall see in the next section, Theorem 1 follows directly from Theorem 2 , whose proof we will concern ourselves with here.

Lemma 2. Using the notations and definitions of Lemma 1, a function $F$ analytic in $D$, with values $F^{(j)}\left(a_{i}\right)=B_{i}^{(j)}$ for $i=1,2, \ldots, m$ and $j=0,1, \ldots, l_{i}-1$, will minimize $\|F\|_{D}$ for all such functions if and only if the function

$$
F_{1}(z)=\left(\frac{1-\bar{a}_{1} z}{z-a_{1}}\right)\left[\frac{\lambda F(z)-\lambda B_{1}^{(0)}}{1-\lambda^{2} B_{1}^{(0)} F(z)}\right],
$$

where $\lambda=1 /\|F\|_{D}$, has the property that if $G(z)$ is analytic in $D$, and $G^{(j)}\left(a_{i}\right)$ $=F_{1}^{(j)}\left(a_{i}\right)$ for $i=1,2, \ldots, m$ and $j=0,1, \ldots, l_{1}-2$ if $i=1$ and $j=0,1, \ldots$, $l_{i}-1$ if $i>1$, then $\left\|F_{1}\right\|_{D} \leqq\|G\|_{D}$.

Proof. Suppose $F^{(j)}\left(a_{i}\right)=B_{i}^{(j)}$ for $i=1,2, \ldots, m$ and $j=0,1, \ldots, l_{i}-1$ and that $\|F\|_{D}$ is a minimum. Let $F_{1}$ be as above. Clearly $F_{1}$ is analytic in $D$ and $\left\|F_{1}\right\|_{D}=1$. If for some other function $G$ analytic in $D$, with $G^{(j)}\left(a_{i}\right)=F_{1}^{(j)}\left(a_{i}\right)$ for $i=1,2, \ldots, m$ and $j=0,1, \ldots, l_{1}-2$ if $i=1$ and $j=0,1, \ldots, l_{i}-1$ for $i>1$, we have $\|G\|_{D}<\left\|F_{1}\right\|_{D}$. Then let

$$
F^{*}(z)=\frac{1}{\lambda}\left[\left(\frac{z-a_{1}}{1-\bar{a}_{1} z}\right) G(z)+\lambda B_{1}^{(0)}\right] /\left[1+\lambda \bar{B}_{1}^{(0)}\left(\frac{z-a_{1}}{1-\bar{a}_{1} z}\right) G(z)\right] .
$$

$F^{*}$ is analytic in $D$ and $\left\|F^{*}\right\|_{D}<\|F\|_{D}$. However,

$$
\begin{aligned}
F(z) & -F^{*}(z) \\
= & \frac{1}{\lambda} \frac{\left(1+\left|\lambda B_{1}^{(0)}\right|^{2}\right)\left(\left(z-a_{1}\right) /\left(1-\bar{a}_{1} z\right)\right)\left[F_{1}(z)-G(z)\right]}{\left[1+\lambda \bar{B}_{1}^{(0)}\left(\left(z-a_{1}\right) /\left(1-\bar{a}_{1} z\right)\right) F_{1}(z)\right]\left[1+\lambda \bar{B}^{(0)}\left(\left(z-a_{1}\right) /\left(1-\bar{a}_{1} z\right)\right) G(z)\right]} \\
= & \phi(z) \prod_{i=1}^{m}\left(z-a_{i}\right)^{l_{i}},
\end{aligned}
$$

where $\phi(z)$ is analytic in $D$. Thus,

$$
F^{*(j)}\left(a_{i}\right)=B_{i}^{(j)} \quad \text { for } i=1,2, \ldots, m \text { and } j=0,1, \ldots, l_{i}-1,
$$

thus contradicting our hypothesis about $F$.

In order to prove the "sufficient" portion, let $F_{1}$ be as defined in the statement of our lemma and let $F_{1}$ have the desired minimizing property. Now suppose $H$ is a function analytic in $D$ with values $H^{(j)}\left(a_{i}\right)=B_{i}^{(j)}$ for $i=1,2, \ldots, m$ and $j=0,1, \ldots, l_{i}-1$, and such that $\|H\|_{D}<\|F\|_{D}$. Let

$$
H_{1}(z)=\left(\frac{1-\bar{a}_{1} z}{z-a_{1}}\right)\left(\frac{\lambda H(z)-\lambda B_{1}^{(0)}}{1-\lambda^{2} \bar{B}_{1}^{(0)} H(z)}\right)
$$

where $\lambda=1 /\|F\|_{D}$. The function $H_{1}$ is thus analytic in $D .\left\|H_{1}\right\|_{D}<\left\|F_{1}\right\|_{D}$ and 


$$
F_{1}(z)-H_{1}(z)=\left(\frac{1-\bar{a}_{1} z}{z-a_{1}}\right)\left[\frac{\left(1-\left|\lambda B_{1}^{(0)}\right|^{2}\right)(F(z)-H(z))}{\left(1-\lambda^{2} \bar{B}_{1}^{(0)} F(z)\right)\left(1-\lambda^{2} \bar{B}_{1}^{(0)} H(z)\right)}\right] .
$$

Thus we see that $H_{1}$ will take on the values $H_{1}^{(j)}\left(a_{i}\right)=F_{1}^{(j)}\left(a_{i}\right)$ for $i=1,2, \ldots$, $m$ and $j=0,1, \ldots, l_{1}-2$ if $i=1$ and $j=0,1, \ldots, l_{i}-1$ if $i>1$. This contradicts our assumption about $F_{1}$, and so our lemma follows.

We will now proceed with the proof of Theorem 2.

Proof of Theorem 2. Let $F$ be analytic in $D$ with values $F^{(j)}\left(a_{i}\right)=f \varphi^{(j)}\left(a_{i}\right)$ for $i=1,2, \ldots, m$ and $j=0,1, \ldots, l_{i}-1$, and suppose $\|F\|_{D}$ is a minimum for all such functions.

Let us first rename the $a_{i}$ 's as follows: let $b_{k}=a_{i}$ for $\sum_{j=1}^{j=i-1} l_{j}<k \leqq \sum_{j=1}^{j=i} l_{j}$. Then define a finite sequence of functions analytic in $D$ by

$$
\begin{aligned}
& F_{0}(z)=\lambda F(z) \quad \text { where } \lambda=1 /\|F\|_{D}, \\
& F_{k}(z)=\left(\frac{1-\bar{b}_{k} z}{z-b_{k}}\right)\left[\frac{F_{k-1}(z)-F_{k-1}\left(b_{k}\right)}{1-\overline{F_{k-1}\left(b_{k}\right)} F_{k-1}(z)}\right],
\end{aligned}
$$

for $k=1,2, \ldots, M$ where $M=\left(\sum_{i=1}^{m} l_{i}\right)-1$. We then have that $\left\|F_{k}\right\|_{D}=1$ for $k=0,1, \ldots, M$; and furthermore, if $G$ is analytic in $D$ and of the form

$$
G(z)=F_{k}(z)+h(z) \prod_{j=k+1}^{j=M+1}\left(z-b_{j}\right),
$$

where $h$ is regular in $D$, then $\left\|F_{k}\right\|_{D} \leqq\|G\|_{D}$. This follows from Lemma 2 . In particular, $\left\|F_{M}\right\|_{D}$ is minimal for all functions $G$ analytic in $D$ which satisfy the single condition $G\left(b_{M+1}\right)=F_{M}\left(b_{M+1}\right)$. It thus follows as a consequence of the maximum principal that $F_{M}$ is identically this constant. Furthermore, by our assumptions we have that

$$
\left|F_{M}\left(b_{M+1}\right)\right|=1
$$

Looking back we find that $F_{M}\left(b_{M+1}\right)$ can be expressed as a rational function in $\lambda$ whose coefficients are determined by the $a_{i}$ 's and the values $f_{Y}^{(j)}\left(a_{i}\right)$, $i=1,2, \ldots, m$ and $j=0,1, \ldots, l_{i}-1$. From (4) we thus obtain a polynomial $\Lambda$ which we can explicitly determine, and of which $\lambda$ is a root, i.e. $\Lambda(\lambda)=0$. The inverse of (2) yields

$$
F_{k-1}(z)=\left[\frac{\left(\left(z-\bar{b}_{k}\right) /\left(1-\bar{b}_{k} z\right)\right) F_{k}(z)+F_{k-1}\left(b_{k}\right)}{1+\bar{F}_{k-1}\left(b_{k}\right)\left(\left(z-\bar{b}_{k}\right) /\left(1-\bar{b}_{k} z\right)\right) F_{k}(z)}\right],
$$

and allows us to calculate $F_{0}$ and then $F$ once we have determined $\lambda$. We note that the values $F_{k-1}\left(b_{k}\right), k=1,2, \ldots, M$, are functions of $\lambda$, and that as defined by (5), each function $F_{k-1}$ will be analytic in $D$ with $\left\|F_{k-1}\right\|_{D}=1$ (as desired) provided that the function $F_{k}$ is and that $\left|F_{k-1}\left(b_{k}\right)\right|<1$. Thus $\lambda$ will be the largest 
positive root of the polynomial $\Lambda$ which satisfies the condition $\left|F_{k-1}\left(b_{k}\right)\right|<1$, for $k=1,2, \ldots, M$. We shall give an example of how $\Lambda, \lambda$ and thus $F$ can be determined later.

From (5) it is also evident that the function $F_{k-1}$ will be rational and $\left\|F_{k-1}\right\|_{D}=1$ for $k=1,2, \ldots, M$, since $F_{M}(z) \equiv \pm 1$. In particular the function $F$ will have the form

$$
F(z)=\frac{1}{\lambda} \prod_{j=1}^{K}\left(\frac{z-c_{j}}{1-\bar{c}_{j} z}\right),
$$

where $\left|c_{j}\right|<1$ for $j=1,2, \ldots, K$ and $K \leqq M=\left(\sum_{i=1}^{M} l_{i}\right)-1$. Note that due to possible cancellation of terms we might have $K<M$.

The uniqueness of $F$ now follows easily. Suppose $G$ is another function analytic in $D$ which assumes the values $G^{(j)}\left(a_{i}\right)=F^{(j)}\left(a_{i}\right)$ for $i=1,2, \ldots, m$ and $j=0,1, \ldots, l_{i}-1$, and such that $\|G\|_{D}=\|F\|_{D}$. Let $\lambda$ be as before and define a sequence $\left\{G_{k}\right\}_{k=1}^{M}$ from $G$ as we did from $F$. We will then conclude that $\left|G_{M}\left(b_{M+1}\right)\right|=1$, and furthermore, we will find that $G_{M}\left(b_{M+1}\right)$ can be expressed as the same rational function of $\lambda$ that we obtained for $F_{M}\left(b_{M+1}\right)$, namely one whose coefficients depend only on the prescribed values of $F$ at the points $\left\{a_{i}\right\}_{i=1}^{m}$. As a result we find that $G_{M}\left(b_{M+1}\right)=F_{M}\left(b_{M+1}\right)$, and then working backwards we obtain $G_{0} \equiv F_{0}$, and thus $G \equiv F$.

Proof of Theorem 1. Let $g$ be as in Corollary 1 , the unique analytic function of "best approximation" to $f$ on $D$. We may then write

$$
\begin{aligned}
\|f-g\|_{U} & =\left\|f_{1}(z)-g(z) \prod_{i=1}^{m}\left(\frac{z-a_{i}}{1-\bar{a}_{i} z}\right)^{l_{i}}\right\|_{D} \\
& \leqq\left\|f_{1}(z)-p_{n}(f, U)(z) \prod_{i=1}^{m}\left(\frac{z-a_{i}}{1-\bar{a}_{i} z}\right)^{l_{i}}\right\|_{D} \\
& =\left\|f-p_{n}(f, U)\right\|_{D}, \quad \text { for } n=0,1,2, \ldots
\end{aligned}
$$

Furthermore, the function $g$ is not only analytic in $D$ but continuous in $\bar{D}=D+U$, and so by Walsh's theorem [4, p.98], given any $\varepsilon>0$, there exists a polynomial $q$ such that $\|g-q\|_{U}<\varepsilon$. Thus for $n$ sufficiently large (greater than the degree of $q$ ), we have

$$
\left\|f-p_{n}(f, U)\right\|_{U} \leqq\|f-q\|_{U} \leqq\|f-g\|_{U}+\varepsilon,
$$

which together with (6) implies that

$$
\lim _{n \rightarrow \infty}\left\|f-p_{n}(f, U)\right\|_{U}=\|f-g\|_{U} .
$$

From (7) and the uniqueness of $g$ it now follows that every subsequence of $\left\{p_{n}(f, U)\right\}_{n=0}^{\infty}$ contains itself, a subsequence that converges uniformly on compact subsets of $D$ to $g$. However, the sequence $\left\{p_{n}(f, U)\right\}_{n=0}^{\infty}$ is equicontinuous on every compact subset of $D$, and so our theorem follows. 
5. An example. We now give a simple application of Theorem 1. Let $f(z)=1 /[z(z-1 / 2)]$. In order to find $\lim _{n \rightarrow \infty}\left[f-p_{n}(f, U)\right]$ we first find that function $F$ analytic in $D$, with values:

$F(0)=f_{1}(0)=1$,

$F(1 / 2)=f_{1}(1 / 2)=4 / 3$, for which $\|F\|_{D}$ is a minimum, where

$f_{1}(z)=1 /(1-z / 2)$.

Let $\lambda=1 /\|F\|_{D}, \quad F_{0}=\lambda F$, and $F_{1}(z)=(1 / z)\left[\left(F_{0}(z)-\lambda\right) /\left(1-\lambda F_{0}(z)\right)\right]$. The function $F_{1}$ must then be a constant, and

$$
\left|F_{1}\left(\frac{1}{2}\right)\right|=\left|2\left[\frac{\lambda((4 / 3)-1)}{1-\lambda^{2}(4 / 3)}\right]\right|=1
$$

In order to determine $\lambda$ we must then find the largest positive root of the quadratic equation $2 \lambda /\left[3\left(1-\lambda^{2}(4 / 3)\right)\right]= \pm 1$ which satisfies $\left|F_{0}(0)\right|<1$ or $\lambda<1$. This yields $\lambda=\left((13)^{1 / 2}-1\right) / 4=.65 \ldots$ Thus $F_{1}(z) \equiv F_{1}(1 / 2)=$ $2 \lambda /\left(3-4 \lambda^{2}\right)=1$, and $F(z)=(1 / \lambda)[(z-\lambda) /(1-\lambda z)]$. It then follows that for $z \in D$,

$$
\lim _{n \rightarrow \infty}\left[f(z)-p_{n}(f, U)(z)\right]\left[\frac{z(z-1 / 2)}{(1-z / 2)}\right]=\frac{1}{\lambda}\left(\frac{z-\lambda}{1-\lambda z}\right)
$$

and so

$$
\lim _{n \rightarrow \infty}\left\|f-p_{n}(f, U)\right\|_{U}=\frac{1}{\lambda}=1.53 \ldots
$$

6. The general case. We now consider the case where $f$ is a function that is continuous on a closed Jordan curve $\Gamma$ and meromorphic inside $\Gamma$.

Letting $\Omega$ denote the interior of $\Gamma$ we have, by Riemann's mapping theorem, that there exists a function $w=\phi(z)$ that is regular in $\Omega$ and maps $\Omega$ conformally onto the open unit disc $D$. Furthermore, since the boundary of $\Omega$ is $\Gamma$, a closed Jordan curve, $\phi$ may be extended continuously. to $\Gamma$ so that it will map $\Gamma$ onto the unit circle $U$ in a one-to-one manner [3, Vol. III, p. 70].

The function $f \circ \phi^{-1}$ will then be meromorphic in $D$ and continuous on $U$. If we let $\left\{\alpha_{1}, \alpha_{2}, \ldots, \alpha_{m}\right\}$ be the poles of $f$ in $\Omega$, then $\left\{a_{1}, a_{2}, \ldots, a_{m}\right\}$ where $a_{i}=\phi\left(\alpha_{i}\right)$ for $i=1,2, \ldots, m$ will be the poles of $f \circ \phi^{-1}$ in $D$. Applying Theorem 1 to $f \circ \phi^{-1}$ on $U$ we have

$$
\begin{gathered}
\lim _{n \rightarrow \infty}\left[f \circ \phi^{-1}(w)-p_{n}\left(f \circ \phi^{-1}, U\right)(w)\right] \prod_{i=1}^{m}\left(\frac{w-a_{i}}{1-\bar{a}_{i} w}\right)^{l_{i}} \\
=\frac{1}{\lambda} \prod_{j=1}^{K}\left(\frac{w-c_{j}}{1-\bar{c}_{j} w}\right) \text { for }|w|<1,
\end{gathered}
$$

where $\lambda, K$ and the $c_{j}$ 's, $j=1,2, \ldots, K$, are as described in Theorem 1, and the convergence is uniform on compact subsets of $D$. 
Theorem 3. Using the above notation,

$$
\begin{array}{r}
\lim _{n \rightarrow \infty}\left[f(z)-p_{n}(f, \Gamma)(z)\right] \prod_{i=1}^{m}\left(\frac{\phi(z)-a_{i}}{1-\bar{a}_{i} \phi(z)}\right)^{l_{i}} \\
=\frac{1}{\lambda} \prod_{j=1}^{K}\left(\frac{\phi(z)-c_{j}}{1-\bar{c}_{j} \phi(z)}\right) \text { for } z \in \Omega,
\end{array}
$$

where the convergence is uniform on compact subsets of $\Omega$.

Proof. Let $P_{n}=p_{n}\left(f \circ \phi^{-1}, U\right), Q_{n}=p_{n}(f, \Gamma)$ for $n=0,1, \ldots$, and $R(w)$ $=\frac{1}{\lambda} \prod_{j=1}^{K}\left(w-c_{j}\right) /\left(1-\bar{c}_{j} w\right)$.

If $z \in \Omega$ and $w=\phi(z)$ we can write

$$
\begin{aligned}
\mid R \circ \phi(z) & -\left[f(z)-Q_{n}(z)\right] \prod_{j=1}^{K}\left(\frac{\phi(z)-a_{i}}{1-\bar{a}_{i} \phi(z)}\right)^{l_{i}} \mid \\
\leqq & \left|R(w)-\left[f \circ \phi^{-1}(w)-P_{n}(w)\right] \prod_{j=1}^{K}\left(\frac{w-a_{i}}{1-\bar{a}_{i} w}\right)\right| \\
& +\left|P_{n}(w)-Q_{n} \circ \phi^{-1}(w)\right| .
\end{aligned}
$$

Consequently, our theorem will follow if it is shown that the sequence $\left\{P_{n}-Q_{n} \circ \phi^{-1}\right\}_{n=0}^{\infty}$ converges to zero uniformly on compact subsets of $D$. In order to accomplish this we first prove that

$$
\lim _{n \rightarrow \infty}\left\|f-Q_{n}\right\|_{\Gamma}=\lim _{n \rightarrow \infty}\left\|f \circ \phi^{-1}-P_{n}\right\|_{U} .
$$

To this end, we observe that since $\phi^{-1}$ is regular in $D$ and continuous in $D+U$, it can be arbitrarily approximated by polynomials on $U$. This follows from Walsh's theorem $\left[4\right.$, p. 98]. Thus given any $\varepsilon_{1}>0$ there exists a polynomial $S_{m}$ such that $\left\|\phi^{-1}-S_{m}\right\|_{U}<\varepsilon_{1}$, and given any $\varepsilon_{2}>0$, by choosing $\varepsilon_{1}$ sufficiently small we will have

$$
\begin{aligned}
\left\|f \circ \phi^{-1}-Q_{n} \circ S_{m}\right\|_{U} & \leqq\left\|f \circ \phi^{-1}-Q_{n} \circ \phi^{-1}\right\|_{U}+\varepsilon_{2} \\
& =\left\|f-Q_{n}\right\|_{\Gamma}+\varepsilon_{2} .
\end{aligned}
$$

But $Q_{n} \circ S_{m}$ is a polynomial of degree $n m$, so we have

$$
\left\|f \circ \phi^{-1}-P_{n m}\right\|_{U} \leqq\left\|f \circ \phi^{-1}-Q_{n} \circ S_{m}\right\|_{U},
$$

which then implies that

$$
\lim _{n \rightarrow \infty}\left\|f \circ \phi^{-1}-P_{n}\right\|_{U} \leqq \lim _{n \rightarrow \infty}\left\|f-Q_{n}\right\|_{\Gamma} .
$$

Similarly given any $\varepsilon_{3}>0$ there exists a polynomial $r_{k}$ such that $\left\|\phi-r_{k}\right\|_{\Gamma}$ $<\varepsilon_{3}$, and given any $\varepsilon_{4}>0$, by choosing $\varepsilon_{3}$ sufficiently small we will have

$$
\left|f-P_{n} \circ r_{k}\right|_{\Gamma} \leqq\left\|f-P_{n} \circ \phi\right\|_{\Gamma}+\varepsilon_{4}=\left\|f \circ \phi^{-1}-P_{n}\right\|_{U}+\varepsilon_{4} .
$$


Repeating our previous argument we obtain (10).

Now let us suppose that on some nonempty compact subset of $D$, $\left\{P_{n}-Q_{n} \circ \phi^{-1}\right\}_{n=0}^{\infty}$ does not uniformly converge to zero. Then since the sequences $\left\{P_{n}\right\}_{n=0}^{\infty}$ and $\left\{Q_{n} \circ \phi^{-1}\right\}_{n=0}^{\infty}$ are equicontinuous on each compact subset of $D$, there exists a subsequence $\left\{P_{k_{n}}-Q_{k_{n}} \circ \phi^{-1}\right\}_{n=0}^{\infty}$, such that:

(i) $\left\{P_{k_{n}}\right\}_{n=0}^{\infty}$ converges uniformly on compact sets of $D$ to a function $L_{1}$ analytic in $D$,

(ii) $\left\{Q_{k_{n}} \circ \phi^{-1}\right\}_{n=0}^{\infty}$ converges uniformly on compact sets of $D$ to a function $L_{2}$ analytic in $D$, and

(iii)

$$
\left\|L_{1}-L_{2}\right\|_{D}>0
$$

By (10) we have

$$
\begin{aligned}
\left\|\left[f \circ \phi^{-1}(w)-L_{1}(w)\right] \prod_{i=1}^{m}\left(\frac{w-a_{i}}{1-\bar{a}_{i} w}\right)^{l_{i}}\right\|_{D} \\
=\left\|\left[f(z)-L_{2} \circ \phi(z)\right] \prod_{i=1}^{m}\left(\frac{\phi(z)-a_{i}}{1-\bar{a}_{i} \phi(z)}\right)^{l_{i}}\right\|_{\Omega} \\
=\left\|\left[f \circ \phi^{-1}(w)-L_{2}(w)\right] \prod_{i=1}^{m}\left(\frac{w-a_{i}}{1-\bar{a}_{i} w}\right)\right\|_{D} .
\end{aligned}
$$

Now by (8),

$$
L_{1}(w)=f \circ \phi^{-1}(w)-R(w) \prod_{i=1}^{m}\left(\frac{1-\bar{a}_{i} w}{w-a_{i}}\right)^{l_{i}} .
$$

Furthermore, by Theorem $1, L_{1}(w)$ is the unique "analytic function of best approximation" to $f \circ \phi^{-1}$ on $D$. This uniqueness together with (13) implies $L_{1} \equiv L_{2}$ and thus our theorem follows.

7. Approximation to arbitrary continuous functions. Although Theorem 3 is not in general applicable for an arbitrary continuous function $f$ on a closed Jordan curve $\Gamma$ (since $f$ is not in general defined in the interior of $\Gamma$ ), a somewhat weaker version can be obtained.

Let $\Gamma$ and $\phi$ be as in $\S 6$. For a function $f$ continuous on $\Gamma$ let

$$
\rho(f, \Gamma)=\lim _{n \rightarrow \infty}\left\|f-p_{n}(f, \Gamma)\right\|_{\Gamma}
$$

Theorem 4. Let $f$ be continuous on $\Gamma$; then given any $\varepsilon>0$ there exists a rational function $R$ of the form

$$
R(w)=\frac{1}{\lambda}\left[\prod_{i=1}^{m}\left(\frac{1-\bar{a}_{i} w}{w-a_{i}}\right)^{l_{i}}\right]\left[\prod_{j=1}^{K}\left(\frac{w-c_{j}}{1-\bar{c}_{j} w}\right)\right],
$$


such that: (i) $\left|\rho(f, \Gamma)-\|R \circ \phi\|_{\Gamma}\right|<\varepsilon$, and

(ii) there exists a polynomial $q$ such that $\|(f-q)-R \circ \phi\|_{\Gamma}<\varepsilon$.

Proof. If $\rho(f, \Gamma)=0$ the theorem is true if we let $R \equiv 0$. Thus we may assume $\rho(f, \Gamma)>0$. It is well known [3, Vol. III, p.100] that if $f$ is continuous on a closed Jordan curve $\Gamma$, then there exists a sequence of rational functions that converge to $f$ uniformly on $\Gamma$. In particular, there exists a rational function $V$ for which $\|f-V\|_{\Gamma}<\varepsilon / 2$. Moreover, $V$ is of the form $V(z)=\sigma(z) / \prod_{i=1}^{m}\left(z-\alpha_{i}\right)^{l_{i}}$, where $\sigma(z)$ is regular on $\Gamma$ and in its interior and the $\alpha_{i}$ 's lie in the interior of $\Gamma$. Since we have assumed that $\rho(f, \Gamma)>0$ it will then follow that $V$ has at least one pole inside $\Gamma$ if $\varepsilon$ is sufficiently small. Let us thus assume that this is the case.

Applying Theorem 3 to $V$ on $\Gamma$, let $R^{*}(z)$ be the limit of the sequence

$$
\left\{\left[V(z)-p_{n}(V, \Gamma)(z)\right] \prod_{i=1}^{m}\left(\frac{\phi(z)-a_{i}}{1-\bar{a}_{i} \phi(z)}\right)^{l_{i}}\right\}_{n=0}^{\infty}
$$

for $z$ in the interior of $\Gamma$, where $\phi$ is as before and $a_{i}=\phi\left(\alpha_{i}\right)$ for $i=1,2, \ldots, m$. The function $R^{*}(z)$ is then of the form

$$
R^{*}(z)=R \circ \phi(z) \prod_{i=1}^{m}\left(\frac{\phi(z)-a_{i}}{1-\bar{a}_{i} \phi(z)}\right)^{l_{i}}
$$

where

$$
R(w)=\frac{1}{\lambda}\left[\prod_{i=1}^{m}\left(\frac{1-\bar{a}_{i} w}{w-a_{i}}\right)^{l_{i}}\right]\left[\prod_{j=1}^{K}\left(\frac{w-c_{j}}{1-\bar{c}_{j} w}\right)\right]
$$

Now for any polynomial $p$ and for $z \in \Gamma$,

$$
\begin{aligned}
|V(z)-p(z)| & \leqq|V(z)-f(z)|+|f(z)-p(z)| \\
& \leqq \varepsilon / 2+|f(z)-p(z)| .
\end{aligned}
$$

Similarly,

$$
|f(z)-p(z)| \leqq \varepsilon / 2+|V(z)-p(z)|
$$

Thus $|\rho(V, \Gamma)-\rho(f, \Gamma)|<\varepsilon$. But $\rho(V, \Gamma)=\|R \circ \phi\|_{\Gamma}$ so (i) follows.

The function $(V-R \circ \phi)$ is continuous on $\Gamma$ and regular interior to $\Gamma$ so again by [4] there exists a polynomial $q$ such that $\|V-(R \circ \phi)-q\|_{\Gamma}<\varepsilon / 2$. Thus,

$$
\begin{aligned}
\|(f-q)-(R \circ \phi)\|_{\Gamma} & \leqq\|f-V\|_{\Gamma}+\|V-(R \circ \phi)-q\|_{\Gamma} \\
& \leqq \varepsilon / 2+\varepsilon / 2=\varepsilon
\end{aligned}
$$


and so (ii) follows.

Remark. If $f$ and $\Gamma$ are as in Theorem 4 , and if one can find a sequence $\left\{R_{n}\right\}_{n=0}^{\infty}$ of rational functions converging to $f$ uniformly on $\Gamma$, and if one can calculate the mapping function $\phi$ for the interior of $\Gamma$, then it is now possible to determine a sequence of polynomials $\left\{q_{k}\right\}_{k=0}^{\infty}$ such that $\lim _{k \rightarrow \infty}\left\|f-q_{k}\right\|_{\Gamma}=\rho(f, \Gamma)$.

\section{REFERENCES}

1. C. Carathéodory and L. Fejér, Rend. Circ. Mat. Palermo 32 (1911), 218-239.

2. G. M.Goluzin, Geometric theory of functions of a complex variable, GITTL, Moscow, 1952; English transl., Transl. Math. Monographs, vol. 26, Amer. Math. Soc., Providence, R.I., 1969. MR 15, 112; MR 40 \#308.

3. A. I. Markuševič, Theory of functions of a complex variable. Vols. I, III, GITTL, Moscow, 1950; English transl., Prentice-Hall, Englewood Cliffs, N.J., 1965, 1967. MR 12, 87; MR 30 \#2125; MR 35 \#6799.

4. G. Meinardus, Approximation of functions: Theory and numerical methods, Springer, Berlin 1964; English transl., Springer Tracts in Natural Philosophy, vol. 13, Springer-Verlag, New York, 1967. MR 31 \# 547; MR 36 \# 571.

5. S. J. Poreda, Estimates for best approximation to rational functions, Trans. Amer. Math. Soc. 159 (1971), 129-135.

Department of Mathematics, Clark University, Worcester, Massachusetts 01610 